\title{
The PSICAT protocol - Primed Subjective-Illusory-Contour Attention Task for studying integrated functional cognitive basis of sustained attention
}

\author{
Benjamin Cowley
}

September 9, 2017

\begin{abstract}
Understanding and measuring sustained attention is important for basic research and applications. Measurement and testing with computerised continuous performance tests is common. When studying the neural correlates of attention, such tests aim to isolate the construct of sustained attention. However, in any ecologically-valid context, sustained attention integrates with other executive functions and depends on lower level perceptual processing. Examination of these interactions is not well-served by existing options, and hence I propose a novel protocol to probe higher cognition and its neural correlates: the Primed Subjective Illusory Contour Attention Task (PSICAT). PSICAT tackles two important qualities of ecological attention: (a) successful attending may require inhibition of interference; (b) natural target stimuli are complex and often noisy. I describe a validation experiment measuring healthy participants with high-resolution electroencephalography. Behavioural and brain-imaging results validate the key hypotheses for the functionality of PSICAT. Additionally, I deomstrate the novel result that task-irrelevant incongruency of an intra-modal distractor prime can evoke stronger behavioural and neural responses than the task-relevant stimulus condition. Finally, the protocol is available as an open-source code repository at the following url, allowing researchers to reuse and adapt it to their particular requirements. https://github.com/zenBen/Kanizsa_Prime/
\end{abstract}




\section{Introduction}

Sustaining attention on task is an important skill for functioning in, e.g. education or the workplace. Sustained attention, also termed vigilant attention, is continuous awareness of a subset of possible stimuli, that is self-directed, task-relevant, and occurs in response to approximately uniform task stimulation (Robertson \& O'Connell, 2010). For example, reading a textbook tends to require sustained attention, whereas reading a flashing advertisement sign is governed by other forms of attention.

Sustained attention is also a skill, and it may be increasingly under threat from modern distractors such as superstimulation (e.g. electronic media) or information overload (e.g. internet addiction) (Newport, 2016). Indeed, clinical pathologies of attention are recognised as a growing problem, in children and adults (Fortenbaugh, DeGutis, \& Esterman, 2017; CHADD, 2017)

Understanding and measuring sustained attention is important for basic research and applications. Measurement and testing is often done with a computerised continuous performance test (CPT), such as the Test Of Variables of Attention (T.O.V.A.). Such 'gold standard' CPTs attempt to index sustained attention by isolating it as a top-down mechanism, using a simple repetitve classification task that strictly controls for cognitive/perceptual complexity by simplifying the stimulus presentation (see Figure 1-a).

However, while such strictly-controlled CPTs may test sustained attention as an isolated construct, it is not isolated in any ecologically-valid context. Sustained attention integrates with other executive functions and depends on lower level perceptual processing. To advance beyond such limited tools and move toward deeper insights into higher cognition and its neural correlates, I present a novel CPT, the Primed Subjective Illusory Contour Attention Task (PSICAT). I also describe a validation experiment measuring healthy participants with high-resolution electroencephalography (EEG). This novel protocol tackles two important qualities of ecological attention: (a) successful attending may require inhibition of interference; (b) natural target stimuli are complex and often noisy.

\subsection{Background}

Existing CPTs work on the principle that sustained attention is an isolable faculty, independent of bottom-up distractor effects, and separate from potential deficits of stimulus processing in the tested modality. However rather

\footnotetext{
${ }^{1}$ Image by Steve Jurvetson, Creative Commons Public Licence (CCPL) https:// creativecommons.org/licenses/by/2.0/legalcode
} 


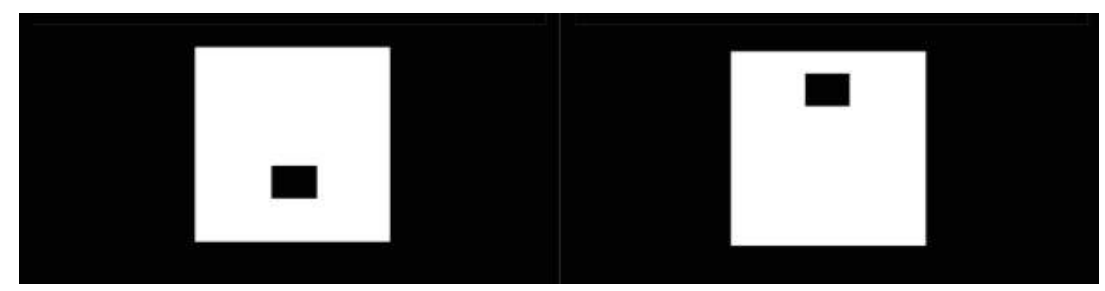

(a)

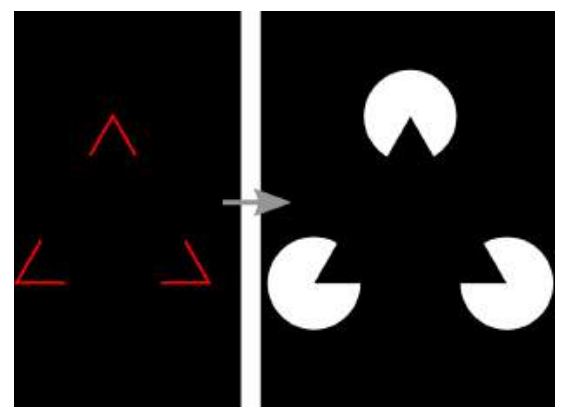

(b)

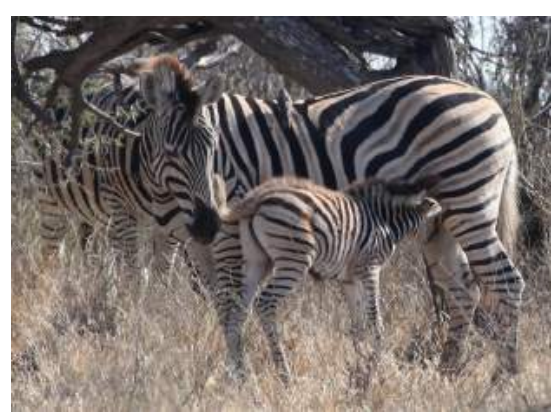

(c)

Figure 1: (a) simple stimuli from the T.O.V.A. test. (b) a triangular Kanizsa subjective contour illusion (right), displayed white-on-black as in PSICAT; with the respective congruent primer (left). (c) "camouflage and camouflage breaking provide an ecological rationale for [gestalt] principles" (Wagemans, Elder, et al., 2012) ${ }^{1}$.

than an single function, sustained attention is a set of cognitive processes which aim to maintain task-focused awareness, specifically: to keep the autonomic nervous system sufficiently aroused, and to orient attention toward the task at hand (Robertson \& O'Connell, 2010). (Clayton, Yeung, \& Cohen Kadosh, 2015) suggested that three kinds of cognitive processes contribute to this:

1. monitor and evaluate ongoing cognitive processes,

2. maintain task-relevant processes through resource allocation,

3. inhibit task-irrelevant processes.

In natural settings any or all of these processes may be impaired in ways that are not dealt with by existing CPTs. Ecological stimuli tend to have compound features, requiring a composition of processes for attending. In visual feature space, natural stimuli are composed of global and local elements, requiring integration (B. Cowley \& Lukander, 2016). Temporally, natural stimuli are related by causal structure, which creates priming effects 
and provides the opportunity for statistical learning. Hierarchically, natural stimuli relate by categorical membership. All these relationships are on display, for example, in Figure 1-c: a herd (global percept), formed of three members of the species Equus quagga (local elements), with changing interactions over time (feeding, fighting, etc), and hierarchical inter-relations (from different perspectives, infant-parent or smaller-larger prey). Task-relevant target stimuli are also commonly accompanied by various forms of intramodal noise, e.g. distortion, occlusion; as well as multi-modal distractors.

In order to provide 'approximately uniform task stimulation' (Robertson \& O'Connell, 2010), a test of sustained attention should not contain extensive temporal structure; in other words, should not evolve any narrative from beginning to end. Respecting this, the PSICAT protocol retains the standard repetitve classification-task structure, and adds complex target stimuli preceded by either congruent or incongruent interference primers. PSICAT thus aims to probe the neural correlates of sustained attention by employing stimuli, primed Kanizsa shapes, which require integrative cognitive processing.

The classification task is based on discrimination of gestalt images from images with identical visual features but no gestalt property. PSICAT uses the Kanizsa subjective contour illusion (SCI; see Figure 1-b), which is a perceived polygon induced by collinear 'Pac-Man' shapes at the vertices.

Kanizsa SCIs are well-studied (see (Banica \& Schwarzkopf, 2016) for a review and see Discussion). As a whole, the neural process of SCI perception has been described as reciprocal, where later visual areas segment the surfaces and assign boundaries needed to perceive illusory objects, and feed the surface/boundary signals back to early visual areas. These signals are then interpreted as an SCI, sometime after the inducer signals are perceived. (Kogo, Strecha, Van Gool, \& Wagemans, 2010) provides a model to describe this complete SCI formation process. This model implies that SCIs cannot be perceived without awareness of the inducing context, i.e. the Pac-Man shapes. In a recent masking-controlled study of the perception of Kanizsa stimuli, (Banica \& Schwarzkopf, 2016) showed strong evidence to support that conclusion. The 'awareness dependence' shown in these works implies that inattention would prevent SCI perception. Based on this, I contend that over many trials Kanizsa presentations should be a good test of sustained attention.

Gestalt imagery are also useful stimuli for testing attention with a more complete evocation of realistic neural processing, for two reasons. Firstly, recognition of the gestalt percept involves the complete visual pathway in the brain (Ringach \& Shapley, 1996). This implies that each trial will comprehensively test the visual attention mode, as opposed to only early areas for recognition of simple shapes. Secondly, gestalt imagery requires conscious 
awareness of the inducing visual features (Banica \& Schwarzkopf, 2016); which implies that over the whole duration the test will validly tap sustained attention (on the condition that participants cannot learn to circumvent the need to recognise the gestalt for discrimination on later trials).

Also, (Marini \& Marzi, 2016) has shown that in event-related potentials (ERPs) of the EEG, early components N1, N2pc, P3a respond to SCIs with larger amplitude than for control stimuli. This suggests that ERP components such as N1 or P3a (both linked to attentional processes (Vogel \& Luck, 2000; Polich, 2007)), can be used to probe participants' processing of SCIs. The effect of sustained attention is also visible in the EEG, as an increase in the fronto-medial (FM) theta-band oscillatory power at the end of the test compared to the beginning (Loo et al., 2009; Juurmaa, 2017).

Finally, Kanizsa images are a good practical choice for protocol design because, despite being a complex percept, are composed of simple geometric forms which facilitates manipulation and control of lower visual features.

\subsection{Summary \& Research Questions}

An experiment is reported herein to validate the ideas and implementation of PSICAT, with research questions as follows. For the following hypotheses I define precise inequalities using abbreviated common terms, which are also used in figures and tables to report results. These include: con congruent; inc incongruent; pri primer stimuli; tgt target stimuli.

Comparing conditions, primer-target incongruence requires inhibition of initial neural response signals, producing longer time to commit a response; on the other hand SCI targets produce faster response times (RTs) than no-SCIs (Tanskanen, Saarinen, Parkkonen, \& Hari, 2008). Meanwhile, the larger early ERPs for SCIs (Marini \& Marzi, 2016) indicate a stronger signal, leading to reduced response time variability (RTV) compared to non-SCI. Thus I have two hypotheses related to response time:

H1rt group mean RTs will be shorter for congruent vs incongruent conditions; and for SCI vs no-SCI conditions moderated by congruency; therefore con.SCI.rt < con.noSCI.rt < inc.SCI.rt < inc.noSCI.rt

H1rtv group mean RTV will be lower for SCI vs no-SCI conditions; therefore SCI.rtv $<$ noSCI.rtv

The priming advantage of congruent conditions implies reduced error rates compared to incongruent. Therefore I have:

H2err error rate will be lower comparing congruent to incongruent conditions; therefore con.error < inc.error 

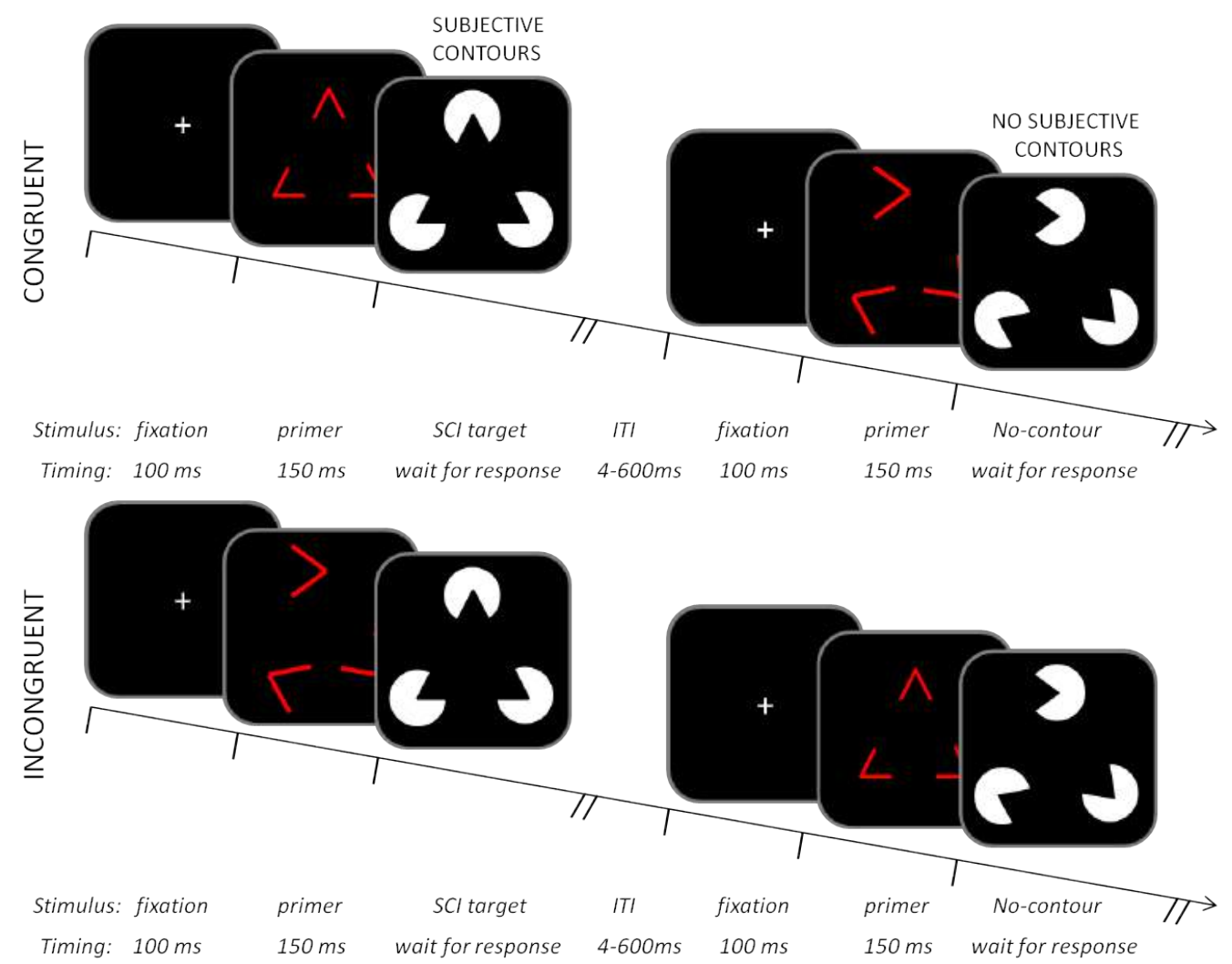

Figure 2: Schematic of single trials in $2 \times 2$ conditions: congruent/incongruent $\times \mathrm{SCI} /$ no-SCI. Congruent primer angles match the following target's angles, incongruent do not match. Trials from each condition are distributed uniformly at random. Participant's task is to classify whether targets are SCI or no-SCI; the primers are to be ignored. PSICAT consists of 550 trials in five blocks, and requires $\sim 22$ minutes to complete.

The neural correlates of PSICAT trials are investigated primarily with ERPs. The largest effect should be due to expectation contradiction: following incongruent trials, the 'novelty' P3b should be larger than similar-target congruent trials. Following (Marini \& Marzi, 2016), N1 (from primers and targets) and P3a (from targets only as P3a from primers would overlap targets) should be larger following SCIs ${ }^{2}$.

Note that due to the two-part nature of the trials (primer and target), the trials are treated as one or two parts depending on the question. Specifically, congruency includes both parts and questions on this will treat a whole trial as a single event; while questions of gestalt shape require looking at primer

\footnotetext{
${ }^{2}$ Since PSICAT is not presented via visual hemifields, N2pc is not tested here.
} 
and target responses separately. Target responses must further be examined separately within congruency conditions, to account for effect of primers. See Methods for practical details. I propose the following ERP-focused hypotheses:

H3P3a P3a will have more positive amplitude for congruent vs incongruent conditions; therefore con.P3a $>$ inc.P $3 a$

H3P3b P3b will have more positive amplitude for incongruent vs congruent conditions; therefore con.P3b $<$ inc. $P 3 b$

H4pN1 In response to primers, visual N1 will have more negative amplitude in SCI than in nonSCI trials; therefore pri.SCI.N1< pri.noSCI.N1

H4tN1 In response to targets (corrected for primer offsets), visual N1 will have more negative amplitude in SCI than in nonSCI trials; therefore tgt.SCI.N1<tgt.noSCI.N1 (in each congruency condition).

H4tP3 In response to targets (corrected for primer offsets), P3a will have more positive amplitude in SCI than in nonSCI trials; therefore tgt.SCI.P3a> tgt.noSCI.P3a (in each congruency condition).

Neural correlates of sustained attention are investigated with FM thetaband power, following (Loo et al., 2009; Juurmaa, 2017). Increased FM theta should be directly attributable to the effect of mental fatigue due to sustained attention, because PSICAT is a context with little to no working memory load. Thus I propose the hypothesis below:

H5fmt FM theta-band power will be greater in the last $10 \%$ of trials compared to the first $10 \%$ of trials; thus fmtheta.first $10 p c<$ fmtheta.last $10 p c$

Finally, primers are attention-grabbing but task-irrelevant, and in a strategic sense should be ignored because only target-recognition determines performance. However the primers are displayed in the same modality as the targets, making them difficult to ignore, and thus their impact will be much larger than their utility to the participant requires. It is thus of basic research interest to examine the relative effect sizes (ES) for tests comparing task-relevant stimulus conditions (SCI vs no-SCI) and congruency - where I predict that congruency effects will be much larger than gestalt-value effects due to their greater adaptive value.

H6es Standardised ES of congruent vs incongruent tests will be larger than standardised ES of SCI vs no-SCI tests; thus es.ConInc > es.SCInoSCI 
In summary, this paper makes three main contributions.

1. The PSICAT protocol provides a well-controlled, novel test of sustained attention, with specific features suitable to probe the components and neural correlates thereof.

2. The protocol code is available online under MIT's open-source licence https://github.com/zenBen/Kanizsa_Prime/, allowing others to use the PSICAT protocol as described, or any part thereof as they see fit.

3. Novel results to H6es show that task-irrelevant incongruency of an intra-modal distractor prime can evoke stronger behavioural and neural responses than the task-relevant stimulus condition.

In the rest of the paper, I first describe the PSICAT features, code and protocol. I then describe the details of a validation experiment testing PSICAT on healthy adult participants. The behavioural and EEG outcomes of this validation are illustrated in the Results section. Finally, I cover the benefits and limitations of PSICAT in the Discussion section, and present my conclusions.

\section{Methods}

\subsection{PSICAT}

The PSICAT protocol has the following novel main features:

1. SCI target trials are contrasted with targets composed of similar PacMan inducers, rotated to disrupt collinearity and prevent inducing a polygon (no-SCI).

2. Targets are preceded by a primer stimulus (see Figure 2), which is either congruent (same inducer angles) or incongruent (different inducer angles) to the target stimulus. Collinearly angled primers do not induce an SCI as strongly as the Pac-Man inducers, but do reinforce the salient features of the following SCI - i.e. the inducer angles. Priming of targets thus manipulates the participant's saliency processing.

3. The protocol randomly generates irregularly-shaped SCIs/no-SCIs (i.e. vertices have arbitrary, though constrained, angles), which prevents participants from learning to classify by minor salient features. For example, in trials with a regular SCI one particular spot on the screen would be occupied by a Pac-Man vertex at a fixed angle; this could be used to discriminate from no-SCIs by focusing on the angle. 
4. The protocol presents both 3- and 4-vertex stimuli, manipulating the quantity of visual information onscreen. This allows separation of the effect of illusory shape (triangle, quadrilateral) from target class (SCI, no-SCI), and also contributes to point 3 above.

5. The open-source code defines stimuli as geometric objects and thus allows users the complete control of parameters: visual angle, timing, luminosity, etc.

The PSICAT protocol thus consists of $2 \times 2$ conditions (of primary interest): congruent SCI, incongruent SCI, congruent no-SCI, incongruent noSCI (Figure 2). A further dimension of vertex number is added for control purposes, giving eight conditions in total; however because there is no taskrelevance to the vertex number it is not a research question and I reasonably exclude vertex number from current analyses.

\subsubsection{PSICAT Presentation code}

In order to create these stimuli with the features described above: irregular shapes, congruent primers, variable vertices; and present them with required randomisation, a comprehensive 'Kanizsa-making' code-base was built for the Neurobs Presentation platform for psychophysics experiments https:// www . neurobs. com.

This code is available from the github repository https://github.com/ zenBen/Kanizsa_Prime/, with comprehensive documentation. The code implements the PSICAT protocol as described, but further allows alteration of PSICAT parameters (timing, conditions), and creation of custom Kanizsa shapes and primers via geometric parameters. The latter enables researchers to develop any form of Presentation experiment with such stimuli.

Briefly, the code consists of two '.sce' files (the Presentation format describing scenarios for experiments), for practice and test versions. There are also five Program Control Language '.pcl' files. PSICAT.pcl defines the particulars of the protocol, such as number of blocks and trials, timing constants, color, etc. This is the code of interest for researchers who want to use the protocol as is, changing only protocol-specific parameters. Three of the other .pcl files contain the functions to generate Kanizsa stimuli. This is the code of interest to users who need such stimuli, whether for use in the PSICAT protocol or another of their own design.

Also in the repository are several images and audio files for the participant's instructions (in Finnish by default but can be localised by the fifth .pcl file). An example experiment file, 'PSICAT.exp', demonstrates how to set up the protocol in Presentation. 


\subsubsection{PSICAT Protocol}

PSICAT is a two-alternative forced-choice task, where the response is a left or right hand button-press to the presence or absence of an SCI in Kanizsa stimuli. Handedness of response is required to be counter-balanced, i.e. randomised across participants.

The protocol begins with an explanation and set of 12 practice trials, three for each condition, to brief participants on the task under supervision of an experimenter. PSICAT consists of five blocks of 110 trials, with a one minute rest break between each block. In each block, trials were uniformly randomly distributed between $2 \times 2 \times 2$ conditions: congruent vs incongruent $\times$ SCI vs no-SCI $\times 3$ - vs 4 -vertex.

Example trials are shown in Figure 2. Each trial consists of a preparatory fixation cross, primer-target pair, and inter-trial interval (ITI). Fixation lasts 100ms; primers are flashed for 150ms; targets are held until the participant responds ( $\sim 750 \mathrm{~ms}$ on average in this study); and ITI is $500 \pm 100 \mathrm{~ms}$, (varied to minimise presentation expectancy which reduces trial effects). Thus an average trial should last $\sim 1.5 \mathrm{sec}$, giving an estimated protocol duration of $\sim 22$ mins, similar to T.O.V.A..

\subsection{Validation experiment}

The operation of PSICAT has been validated by deployment in a clinical trial (B. Cowley, Holmström, Juurmaa, Kovarskis, \& Krause, 2016), where it was used to test adult ADHD participants alongside the healthy participants who are reported herein (the clinical results are to be reported elsewhere; this context is mentioned merely for full disclosure).

\subsubsection{Participants}

17 neurologically healthy participants were recruited by word of mouth, and were remunerated. They had normal or corrected-to-normal vision, were all right-handed, and were not taking any medication. There were 11 females, 6 males; average age was 34 (standard deviation 11). Participants provided written informed consent before entering the study. The protocol followed the Declaration of Helsinki, and ethical approval was obtained from The Ethical Committee of the Hospital District of Helsinki and Uusimaa, 28/03/2012, 621/1999, $24 \S$. 


\subsubsection{EEG recording}

In an electrically shielded room, EEG data was continuously recorded (DC$104 \mathrm{~Hz}$; sampling rate (SR) $512 \mathrm{~Hz}$ ) using Biosemi ActiveTwo amplifier, with 128 active electrodes mounted in a fitted flexible cloth cap according to the Biosemi 128 montage. Offline reference electrodes were attached at the mastoids. Electrooculography was measured with two bipolar electrode sets, mounted horizontally at the outer canthi of each eye, and vertically above and below the left eye.

Additionally, electrocardiography was measured from chest-mounted electrodes at the manubrium and lower left rib. Electrodermal activity was measured from the proximal phallanges of the index and forefinger on the nondominant hand. These autonomic nervous system signals are not analysed herein.

Biosemi ActiveTwo equipment reduces the need for impedance measurement $^{3}$. Instead, the quality of contact between electrode and skin was monitored using running average voltage offset at each electrode, which was kept below $\pm 25 \mathrm{mV}$.

\subsubsection{Procedure}

Participants were tested with PSICAT as the second in a battery of tests. The whole session consisted of: briefing, dressing in the electrodes, passive baseline measurement (4 minutes), T.O.V.A. CPT (22 minutes), PSICAT protocol ( $\sim 22$ minutes), eyes-closed vigilance protocol ( $\sim 15$ minutes), and post-test baseline (4 minutes). The recording period lasted just over one hour.

\section{$2.3 \quad$ Analysis}

\subsubsection{Data preprocessing}

Behavioural data was obtained from the Presentation log file, and analysed in the R platform for statistical computing (R Core Team, 2014).

EEG data was imported to Matlab (Natick, MA) using the EEGLAB toolbox (Delorme et al., 2011). The data was preprocessed using batch scripts powered by CTAP (B. U. Cowley, Korpela, \& Torniainen, 2017), in the following steps:

- Highpass filtering at $0.5 \mathrm{~Hz}$, using Matlab's least-squares linear-phase finite impulse response (FIR) filter design, with filter order of $1.5 \times \mathrm{SR}$,

\footnotetext{
${ }^{3}$ see http://www.biosemi.com/faq/cms\&drl.htm
} 
frequency characteristics $\left[0 \frac{0.85}{S R} \frac{1}{S R} 1\right]$, and amplitude characteristics [0 $\left.\begin{array}{lll}0 & 1 & 1\end{array}\right]$.

- Bad channel detection by the union of: visual identification made during the recording session, and automated classification using the FASTER toolbox (Nolan, Whelan, \& Reilly, 2010). Detected channels were removed (per participant $M=3.6, S D=2.2$ ).

- Bad epoch detection by automated classification with the FASTER toolbox (Nolan et al., 2010). Detected epochs were removed (per participant $M=19, S D=5)$.

- Independent Components (IC) Analysis using the extended Infomax algorithm after (Lee, Girolami, \& Sejnowski, 1999).

- Bad IC detection using the union of FASTER (Nolan et al., 2010) and ADJUST (Mognon, Jovicich, Bruzzone, \& Buiatti, 2011) automated classifcation. Detected ICs were removed and their activations subtracted from the EEG data using EEGLAB's pop_subcomp () function.

- Spherical interpolation of rejected channels.

\subsubsection{EEG analysis}

ERPs were generated from the EEG data using Matlab. Continuous EEG was split into $750 \mathrm{~ms}$ epochs, with $100 \mathrm{~ms}$ pre-stimulus baseline, primer lasting $150 \mathrm{~ms}$, and $500 \mathrm{~ms}$ after target stimulus onset. Epochs were baselinecorrected with respect to the mean voltage of the $100 \mathrm{~ms}$ period preceding the part of the epoch relevant to the research question. Specifically, for H3P3a,b and H4eap the baseline was $-250 \ldots-150 \mathrm{~ms}$, while for H4eat it was $-100 \ldots 0 \mathrm{~ms}$ (i.e. the latter $2 / 3 \mathrm{~s}$ of the primer presentation). After baseline-correction, a $20 \mathrm{~Hz}$ low-pass filter was applied for visualisation and testing.

To address H3P3a,b, P3a and P3b curves were generated for ROIs chosen based on (Polich, 2007): for P3a, the vertex plus five closest electrodes (A2 B1 C1 D1 D15); and for P3b, midline parietal electrode A19 (equivalent to $\mathrm{Pz}$ under 10/20 system) plus four closest electrodes (A4 A5 A20 A32). See Figure 4 for ROI locations. These ERPs were averaged in congruent and incongruent conditions separately.

To address $\mathbf{H} 4 \mathbf{p N 1}$, tN1,tP3, curves for early components were generated at a region of interest (ROI) centered on A23 (equivalent to Oz under 10/20 system) plus A15 A22 A24 A28 (i.e. four closest electrodes). This ROI was chosen to broadly reflect all early visual-area activity. 
To address H5fmt, epoched EEG data was subsetted to the first $10 \%$ and last $10 \%$ of epochs (10\% was chosen to emulate the approach in (Loo et al., 2009)). Spectral power was estimated within each of these early and late subsets for all trials (i.e. pooling all conditions), and compared by two separate methods. First, the Fast Fourier Transform (FFT) estimate of spectral power was computed (using EEGLAB's pwelch() method) for the region of interest $\mathrm{C} 19-\mathrm{C} 21$ (C20 = Fz in the 10/20 system, plus adjacent midline electrodes). Second, event-related spectral perturbation (ERSP) was computed at $\mathrm{C} 20$ alone (using the timef () method implemented in EEGLAB). ERSPs measure signal power relative to a baseline, expressed in decibels, and so were plotted and tested against the average baseline of combined early and late subsets, to control for signal drift from beginning to end of the CPT. Due to the constraints of the epoch size and the desired frequency range (i.e. lower frequencies cannot be calculated without having more 'pad' data around the epoch of interest), ERSP duration was limited to $0 \ldots 300 \mathrm{~ms}$. The FFT shows the absolute difference in spectral power at every frequency, thus supporting interpretation of the ERSP, which only shows relative power but reveals the temporal dynamics. Lastly, the inter-trial coherence (ITC) measure of stimulus-onset phase locking was estimated at C20 for the same time range as ERSP, to assess phase coherence across trials (Delorme \& Makeig, 2004). ITC has been positively associated with relative levels of attending to a trial-wise targets (Yamagishi, Callan, Anderson, \& Kawato, 2008), and thus should help clarify the effect of FM theta power changes.

\subsubsection{Statistical methods}

For testing H1rt and H1rtv I first removed error trials. For H1rt I analysed participant-wise median RTs in order to control outliers and obtain a robust central tendency estimate (Whelan, 2010). Although still a biased estimator, condition-bias will be approximately equal when the number of trials per condition is equal, which is true here due to the low error rates. For H1rtv I analysed participant-wise variability of RTs.

The paired samples for each comparison were tested for homogeneity of variance by Levene's test, passing in all cases. Samples were all shown to be strongly non-normal by Shapiro-Wilk test, indicating a non-parametric test. Condition differences were thus tested by pairwise one-sided Mann-Whitney U-tests. ESs for these tests was calculated by $E S=z \frac{1}{\sqrt{N}}$, where $z$ is the test z-score and $N$ is total number of observations (after (Field, 2009, pp.550)).

ERP amplitude was estimated as the mean across a window, instead of selecting a peak value, as recommended by Luck (Luck, 2014). Window length was fixed proportional to the component duration, i.e. $20 \mathrm{~ms}$ for N1 
and 40ms for P3a,b. Window latency was centered on the mean peak latency of the relevant grand average ERPs. Thus condition- and participant-wise mean amplitude was calculated within windows: primer N1 $0 \pm 10 \mathrm{~ms}$; target N1 165 $\pm 10 \mathrm{~ms}$; P3a $280 \pm 20 \mathrm{~ms}$; P3b $480 \pm 20 \mathrm{~ms}$. The resulting values were tested for condition-wise difference using paired-sample one-tailed $t$-test. Cohen's $d$ ES was computed for these results using the 'compute-es' package for R platform (Re, 2013).

To estimate the statistical difference of FM theta-band power in first and last $10 \%$ of trials, I used EEGLAB's permutation testing procedure. This constructs a distribution for the null-hypothesis of no difference in conditions, and conducts an (unpaired) test for whether the observed value lies in the tails of the distribution. This procedure was applied (using EEGLAB) for power spectrum, ERSP, and ITC comparison. Resulting significant frequencies $\times$ times are indicated in plots accompanying those data visualisations. Permutation testing gives stochastic output for exact regions of significance, so calculations were repeated ten times to ensure that what is displayed is characteristic of a repeatable pattern.

Finally, to test whether ESs from above results were larger for congruency than for (no-)SCI questions, I compared the pooled absolute values of ESs from significant results, separately for behavioural and neural data sources (avoiding mixing ES-estimation methods). This gave the following (no-)SCI vs congruency comparisons: H1rtv vs H2err; [H4pN1, H4tN1, H4tP3a] vs $[\mathbf{H 3 P 3 a}, \mathbf{H 3 P 3 b}$. To compare, I took the median ES value and substituted the nominal 'rule-of-thumb' value (small, medium, large, extended according to (Sawilowsky, 2009) for Cohen's $d$ ) to demonstrate the inequality. Because ES values are standardised, there was no need to do further statistical testing.

For each family of analyses, family-wise error rate was controlled by $p$ value adjustment using the Holm-Bonferroni method.

\section{Results}

\subsection{Behavioural Results}

Condition RTs were ordered as expected con.SCI.rt $<$ con.noSCI.rt $<$ inc.SCI.rt < inc.noSCI.rt, as shown in Figure 3 (note that these relationships are transitive). Further, condition of target SCI versus no-SCI followed the predicted inequality for RTV, SCI.rtv < noSCI.rtv; as did condition of congruent versus incongruent trials for errors, con.err $<$ inc.err. All condition differences of behaviour were significant (after multiplicity adjustment) 
for each predicted inequality as shown in Table 1, indicating support for H1rt, H1rtv, and H2err.

Table 1: Hypothesis test results for H1rt, H1rtv, and H2err. Columns are: Hyp. Hypothesis; Condition comparison; Med. diff median difference of conditions; U Mann-Whitney U-test statistic; adj. p Bonferroni-Holm adjusted $p$-values; Sig. significance level (as below); ES effect size.

$\cdot p \leq 0.1,{ }^{*} p \leq 0.05,{ }^{* *} p \leq 0.01,{ }^{* * *} p \leq 0.001,{ }^{* * * *} p \leq 0.0001$

\begin{tabular}{llccccc}
\hline Hyp. & Condition comparison & Med. diff & $U$ & adj. $p$ & Sig. & ES \\
\hline H1rt & con.SCI.rt < con.noSCI.rt & $51 \mathrm{~ms}$ & 0 & $4 \times 10^{-5}$ & $* * * *$ & 0.99 \\
H1rt & con.noSCI.rt < inc.SCI.rt & $19 \mathrm{~ms}$ & 38 & 0.04 & $*$ & 0.51 \\
H1rt & inc.SCI.rt < inc.noSCI.rt & $22 \mathrm{~ms}$ & 20 & 0.008 & $* *$ & 0.64 \\
H1rtv & SCI.rtv < noSCI.rtv & $14 \mathrm{~ms}$ & 161 & 0.02 & $*$ & 0.57 \\
H2err & con.err < inc.err & $1.1 \%$ & 1 & $6 \times 10^{-5}$ & $* * * *$ & 0.97 \\
\hline
\end{tabular}

\section{$3.2 \quad$ Neural Results}

Table 2 shows the results of ERP tests for hypothesis sets 3 and 4 .

Table 2: Significance tests for the condition-wise difference of mean amplitude $(\mu \bar{V})$ within time windows corresponding to ERP components. Columns are: Hyp. hypothesis; Cnd. congruency condition (only for tests of SCI vs no-SCI); ERP tested component; ROI centre of region of interest; Diff $\mu \bar{V}$ condition-wise difference of mean amplitude in microvolts; $\mathbf{t}$ t-statistic; $\mathbf{d f}$ degrees of freedom; adj. p Bonferroni-Holm adjusted p-value; Sig. significance level (as below); ES Cohen's $d$ effect size.

$\cdot p \leq 0.1,{ }^{*} p \leq 0.05,{ }^{* *} p \leq 0.01,{ }^{* * *} p \leq 0.001,{ }^{* * * *} p \leq 0.0001$

\begin{tabular}{llllllllll}
\hline Hyp. & Cnd. & ERP & ROI & Diff $\mu V$ & $t$ & df & adj. $p$ & Sig. & ES \\
\hline H3P3a & - & P3a & vertex & 3.23 & 6.68 & 33 & $5 \times 10^{-7}$ & $* * * *$ & 2.29 \\
H3P3b & - & P3b & parietal & -3.09 & -5.67 & 33 & $8 \times 10^{-6}$ & $* * * *$ & -1.94 \\
H4pN1 & pri & N1 & occipital & -1.30 & -4.49 & 33 & $2 \times 10^{-4}$ & $* * *$ & -1.54 \\
H4tN1 & con & N1 & occipital & 1.67 & 3.54 & 16 & 0.99 & ns & - \\
H4tN1 & inc & N1 & occipital & -1.59 & -3.06 & 16 & 0.01 & $*$ & -1.05 \\
H4tP3a & con & P3a & vertex & 1.63 & 2.29 & 16 & 0.05 & $*$ & 0.79 \\
H4tP3a & inc & P3a & vertex & 1.00 & 1.76 & 16 & 0.09 &. & 0.6 \\
\hline
\end{tabular}

The significance testing shows strong support for H3P3a and H3P3b. The P3a recorded at the vertex ROI, and P3b at centro-parietal ROI, are 


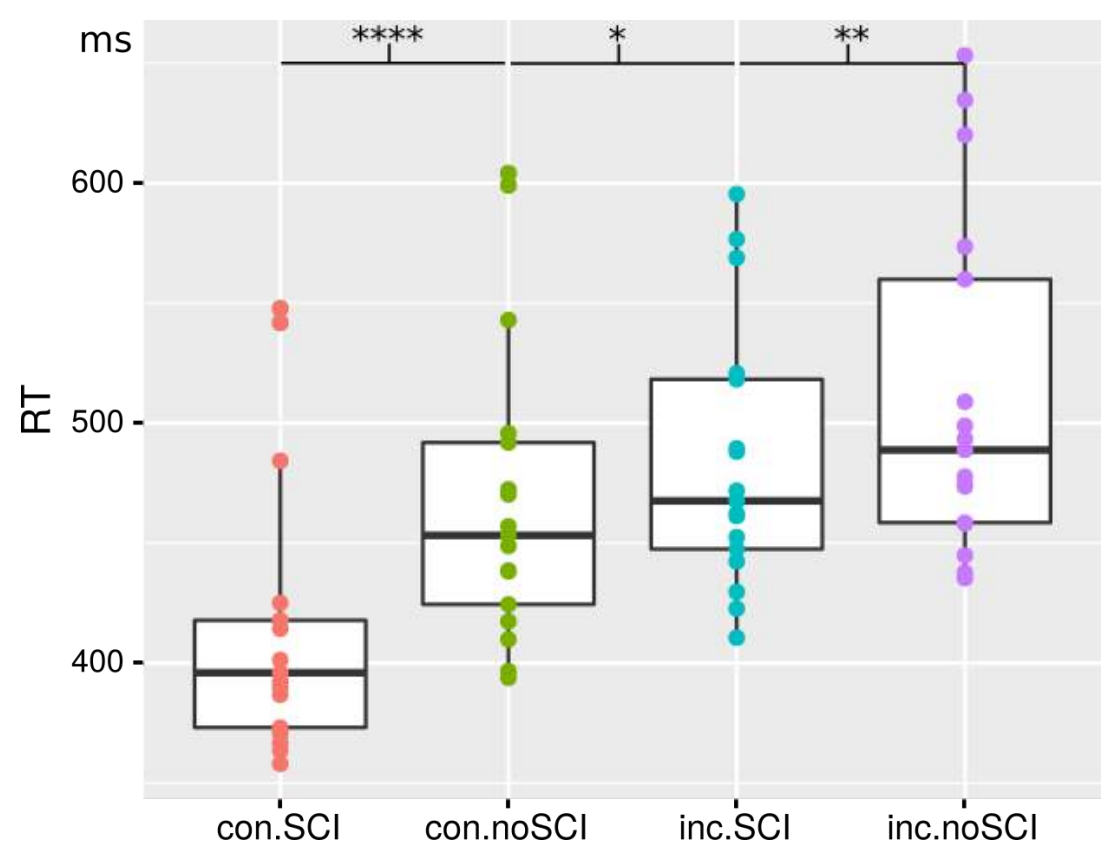

Figure 3: RTs in ms for hit trials in each primary condition (L to R: congruent SCI, congruent no-SCI, incongruent SCI, incongruent no-SCI); points are median RTs per participant; boxes show: sample median, first and third quartiles, and whiskers at $1.5 \times$ inter-quartile range. There is a clear condition-wise trend, with decrease of speed following congruency and then SCI.

shown in Figure 4 (panel A and B, respectively). Both panels also show the associated scalp maps for the time window of testing, indicating the spatial activation during P3a,b and the context of the ROIs.

The tests also show partial support for the relatively greater effect of SCIs on early components. $\mathbf{H} \mathbf{4 p N} \mathbf{1}$ is strongly supported so primer-condition is definitely relevant to the processing of subsequent targets. $\mathbf{H 4 t N 1}$ is supported but only for the incongruent condition. The congruent condition difference is similarly large but has opposite sign to what was expected, thus non-significant under one-tailed test. The test for H4tP3a was significant for congruent condition, and marginal for incongruent, but with only small effect size. Early components are illustrated in Figure 5 below.

Examining the FM theta-band power to test the effect of sustained attention, H5fmt is supported, theta-band power significantly higher in late than early trials by FFT analysis, $p<0.01$; and significantly reduced trial-wise divergence of theta power by ERSP analysis, $p<0.05$. The phase-locking analysis also shows significantly lower ITC for late than early trials in thetaband, $p<0.01$. 


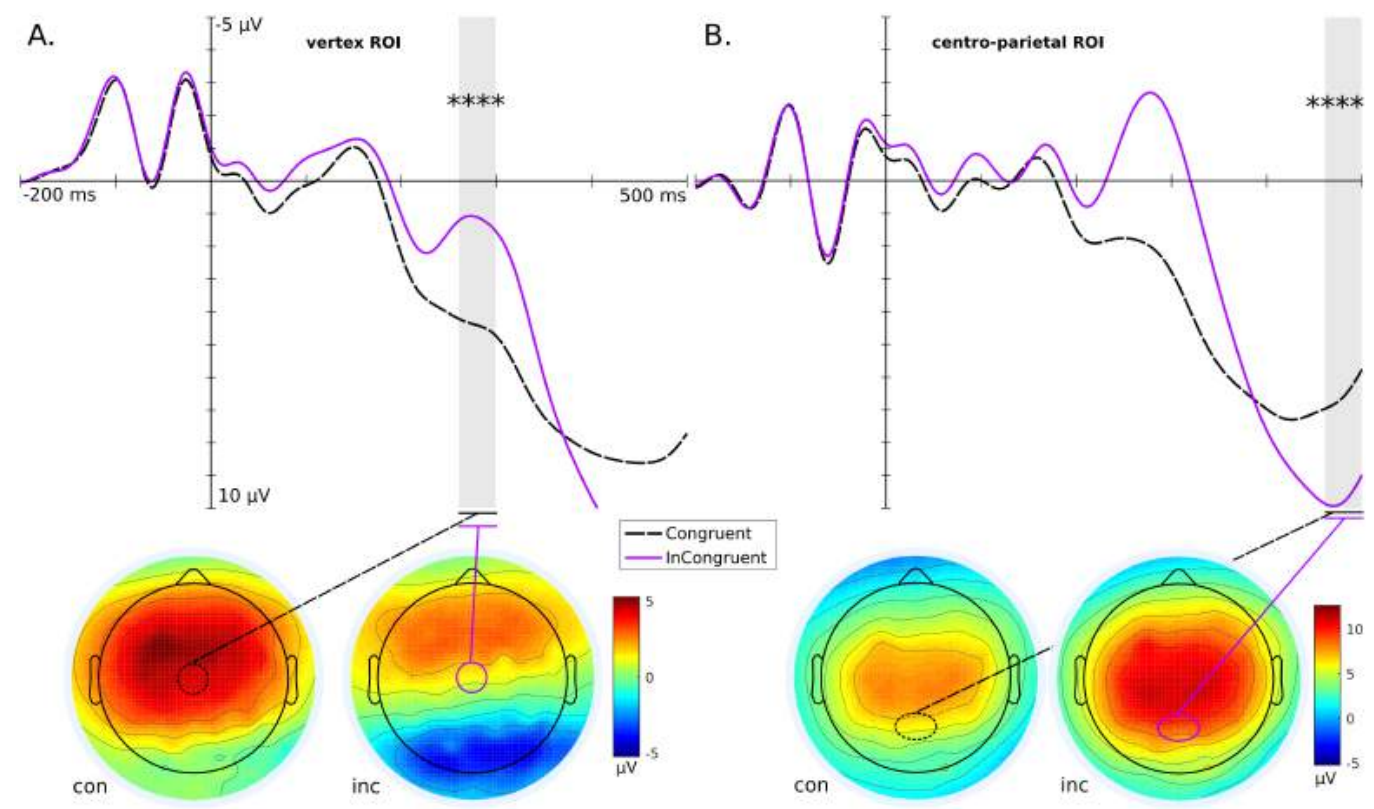

Figure 4: Spatial and temporal activations in congruent and incongruent conditions shown by P300 grand average ERPs (calculated for ROIs) and associated whole-head scalp maps (calculated for the time window of curve testing) to show the spatial extent of the ROI. Significance levels: **** $p \leq 0.0001$ Panel A: P3a at the vertex ROI, significantly larger amplitude for congruent than incongruent within the grey highlighted time-window, with condition-wise scalp maps. Panel B: P3b at the centro-parietal ROI, significantly larger amplitude for incongruent than congruent within the grey highlight, with condition-wise scalp maps.

Finally, H6es is supported by larger ESs for congruency than for (no-)SCI questions, as shown below in Table 3 .

\section{Discussion}

Results have broadly validated PSICAT in its designed purpose, and shown novel effects relating interference inhibition to gestalt processing. Below I discuss behavioural and neural results in turn, but first describe the relevant state of the art on gestalt image studies. 


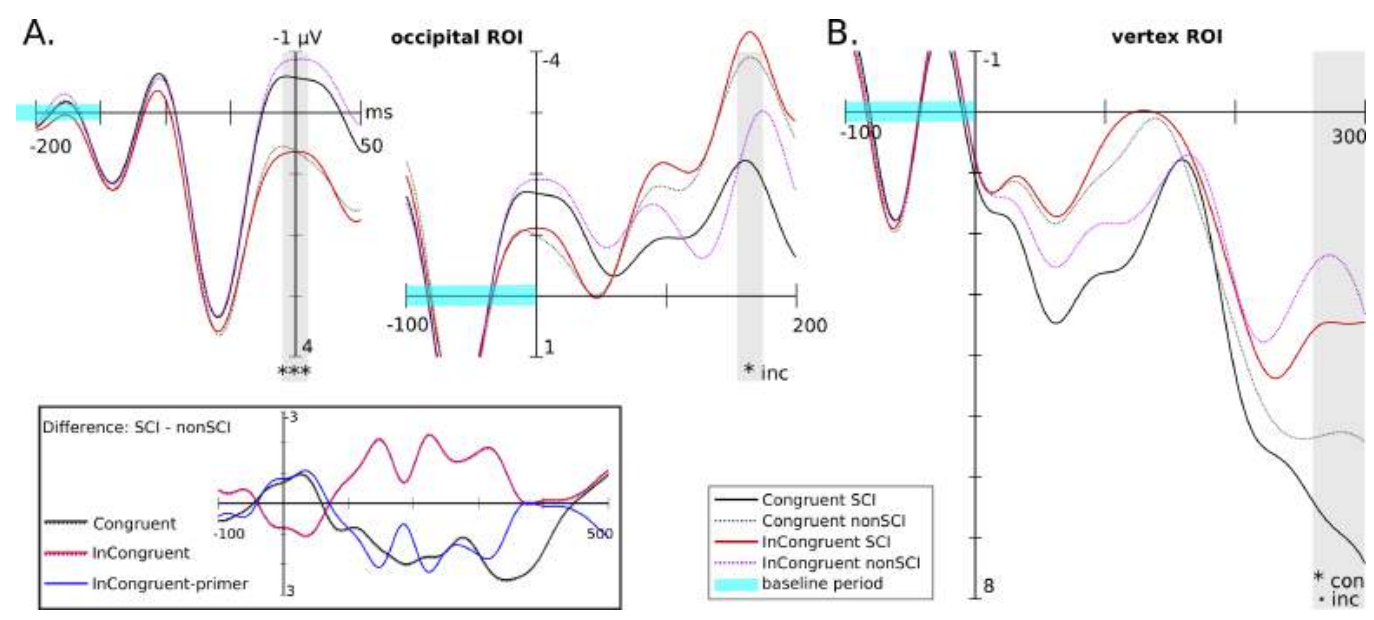

Figure 5: Early components in response to gestalt/non-gestalt stimulus conditions shown by grand average ERPs calculated over ROIs. Significance levels: $\cdot p \leq 0.1,{ }^{*} p \leq 0.05,{ }^{* * *} p \leq 0.001$ Panel A: N1 at the occipital ROI for primer (left) and target (right) (note each plot uses different baseline period). To clarify the relation between conditions, the inset box shows the difference wave across the whole target period. N1 has significantly larger amplitude for SCI than non-SCI within the grey highlighted time-windows, in response to primers and congruent targets, but the pattern is reversed for incongruent targets. Panel B: P3a at the vertex ROI, showing significantly larger amplitude for SCI than non-SCI within the grey highlight (marginal for incongruent).

\subsection{Gestalt imagery}

Gestalts have been well-studied in the literature (dating back over 100 years with a recent resurgence of interest), investigated both visually and cognitively. In a comprehensive review, (Wagemans, Elder, et al., 2012; Wagemans, Feldman, et al., 2012) describe the conceptual and theoretical foundations of the gestalt approach to perceptual science, including implications for attention.

(Tanskanen et al., 2008) demonstrate that stimuli with global contours are processed differently to those with equivalent local visual features but no contours, in later visual areas during $130 \ldots 300 \mathrm{~ms}$. The most pronounced and earliest difference was for a stimulus with a gestalt property where global contours are integrated to a single whole, which consequently showed the fastest reaction times.

In agreement with (Tanskanen et al., 2008), a study by (Harris, Schwarzkopf, Song, Bahrami, \& Rees, 2011) demonstrated that the Kanizsa SCI percept 

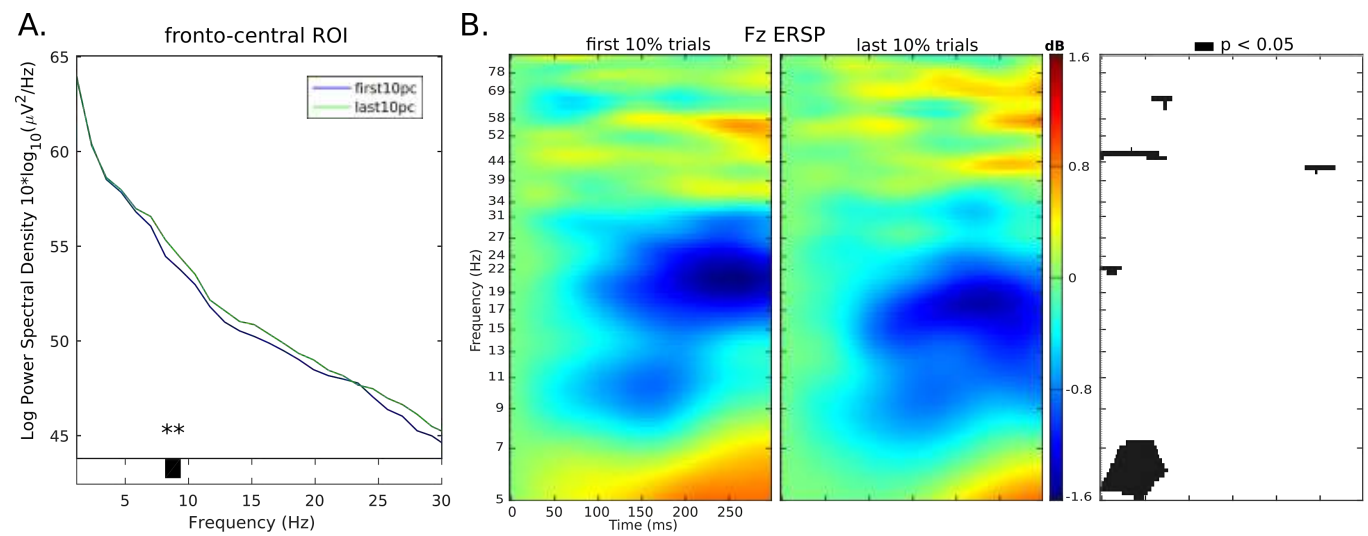

C.

D.
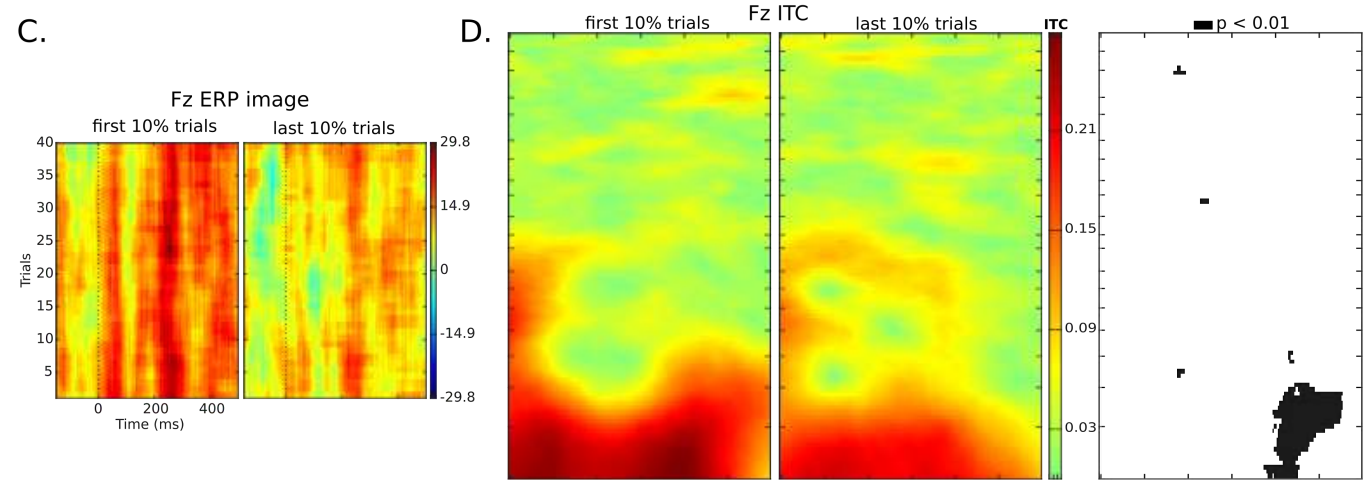

Figure 6: Spectral power comparisons between first $10 \%$ and last $10 \%$ of trials, at fronto-medial electrodes. Panel A: The FFT power spectrum from 1-30 Hz shows that FM theta band power is significantly higher in the late than early subset of trials (by permutation testing). Panel B: The ERSP indicates reduced divergence from baseline power in late trials, significant in theta-band at $0 \ldots 100 \mathrm{~ms}$. Correspondence of the significant frequencies in FFT and ERSP analyses suggests that relative trial-wise power increases (in ERSP) are reduced due to a higher overall level of power. Panel C: The ERP image of early and late subsets, showing the relatively greater positivities in early trials. No statistical testing was done as this image is for exploratory purposes. Panel D: The ITC of early and late subsets shows relative reduction in phase-locking in later trials, significant in the theta and low-alpha bands at $\sim 200 \ldots 300 \mathrm{~ms}$.

is generated at a later stage of visual processing. This was shown by participants' inability to discriminate orientation of a Kanizsa triangle SCI when the inducers (Pac-Men) were masked from attention; whereas using the same method to mask the context of a simultaneous brightness contrast illusion did not affect the illusory percept. More evidence came from (Murray et al., 
Table 3: The nominal comparison of effect sizes for significant results pertaining to questions of (no-)SCI compared to questions of congruency.

\begin{tabular}{lll}
\hline Result comparison & Med. ES & Nominal ES \\
\hline H1rtv vs H2err & 0.57 vs 0.97 & medium vs large \\
(H4pN1,H4tN1,H4tP3a) vs $(\mathrm{H} 3 \mathrm{P} 3 \mathrm{H}, \mathrm{H} 3 \mathrm{P} 3 \mathrm{~b})$ & 1.05 vs 2.115 & large vs huge \\
\hline
\end{tabular}

2002), who proposed that SCI sensitivity in early visual areas V2 and V1 is mainly due to feedback modulation from higher areas. Indeed, Kanizsa SCIs have been shown to reliably activate the higher extrastriate cortex even when manipulation of visual features, such as rounding corners of the PacMan inducers, reduced the SCI percept strength (Ringach \& Shapley, 1996).

On the other hand, the SCI perception is not merely top-down classification, as illusory contours have been shown to be processed by binocular neurons in early visual cortex (Häkkinen \& Nyman, 2001).

As a whole, the process of SCI perception has been described as reciprocal, where later visual areas segment the surfaces and assign boundaries needed to perceive illusory objects, and feed the surface/boundary signals back to early visual areas. These signals are then interpreted as an SCI, sometime after the inducer signals are perceived. The model by Kogo et al (Kogo et al., 2010) describes this complete SCI formation process. This model implies that SCIs cannot be perceived without awareness of the inducing context, which is empirically supported by Banica et al (Banica \& Schwarzkopf, 2016).

Additionally, the neural signature should betray whether the SCI was 'invisible' due to inattention. Schurger et al (Schurger, Sarigiannidis, Naccache, Sitt, \& Dehaene, 2015) show that repeated stimulus presentation creates reduced variability in post-stimulus cortical activation, predictive of whether the stimulus was subjectively seen, suggesting that "conscious perception may involve the transient stabilization of distributed cortical networks, corresponding to a global brain-scale decision." This suggests that the neural signature of trials should differ if the participant actually perceives the SCI or not, potentially providing a more accurate measure than behavioural responses (given that chance level of responding is 50\%). Isolating the relevant global neural signal for analysis is another matter (which is why this question has been left as future work).

Another implication of the (Kogo et al., 2010) model affects the PSICAT primers: because SCI formation is relatively slow, the primer has small chance to induce SCI. (Ringach \& Shapley, 1996)'s results on reduction of SCI perception strength due to alteration of inducer visual properties also supports the notion that the primers, being not complete Pac-Man induc- 
ers, will not easily induce SCI. Thus, primers should play the role to prime participants to expect particular classes of Kanizsa shapes (SCI or no-SCI) without forming a 'false-positive' SCI. (Schwarzkopf \& Rees, 2011) has previously illustrated that response times were faster when gestalt targets were preceded with shape-congruent primes.

(Wasserstein, Zappulla, Rosen, Gerstman, \& Rock, 1987) investigated four perceptual closure tests, including SCIs, in a sample of focal lesion patients; they found that SCI processing was right-hemisphere dependent.

\subsection{Behavioural Results}

Natural visual attention, e.g. scanning the Figure 1-c, requires processing of hierarchies both at input, (compositing scenes from low-level features), and at representation (attention can be directed to the global or local level). The integrated functioning of global and local processing is important for object recognition and contextualisation (Hellige, 1993, p.75). One aspect of integration is feature saliency, which refers to an order of importance attached to features of the scene by the visual attention system, making certain features 'stand out'. Order of processing (for sudden-onset stimuli) is $\sim 25-50 \mathrm{~ms}$. Task-related or top-down visual attention can modulate attention but the order is much slower, $\sim 200 \mathrm{~ms}$ or more (Itti \& Koch, 2001).

The Kanizsa imagery allows both classes of primer and target stimuli, SCI or no-SCI, to have roughly equal spatial and luminance frequency characteristics. To distinguish between them thus depends more on the implicit shape than on the visual characteristics of the target itself, implying that the visual salience feature of implicit shape (Wolfe \& Horowitz, 2004) guides attention.

Thus, the results for response time and error-rate generally confirm the expectations regarding stimulus and congruency condition effects. Namely, congruent trial responses are faster and less error-prone than incongruent trials; while SCI targets are faster and more consistent than no-SCI targets.

As noted above, the condition RT inequalities (Figure 3) are transitive. Thus condition con.SCI not only has shorter RTs than the condition it is tested against, con.noSCI, but each other condition too. This implies that the comparison of pooled conditions can be inferred, i.e. congruent RTs are (statistically significantly) less than incongruent, and inc.noSCI RTs are greater than all other conditions combined. The latter implies that the effect of an SCI primer magnifies the effect of incongruency, in addition to obviously enlarging the speed of recognition of an SCI target.

Behavioural results thus seems to validate the design of PSICAT. Additionally, though not a research question, the error rate is observed to be 
low at $1 \ldots 3 \%$, with similar variability. This is because the protocol allows participant-paced responding, which improves the power of the test to probe neural responses to hit trials, but limits the chance to study error-related activations. Target duration is a trivial matter to change in the PSICAT code, but if targets are set to fixed durations, it is likely the experimenter should increase the duration of ITIs to prevent activation overlap between trials for slow responders.

\subsection{Neural Results}

Various methods have been used to examine the neural correlates of sustained attention from electrophysiology data. In the time domain these include, e.g. event-related potentials (ERPs) (Woodman, 2010) and phase dynamics (Palva \& Palva, 2007); and in frequency domain there are, e.g. eventrelated spectral perturbations (ERSPs) and frequency-band power (B. Cowley, Filetti, et al., 2016).

\subsubsection{Neural correlates of trial-wise attention}

The primary method used to examine neural correlates of the PSICAT trials are ERPs. I primarily followed recent work that showed enhanced attention capture by gestalt stimuli, with greater amplitude for early components N1, N2pc and P3a (though no difference for P1) compared to matched control stimuli (Marini \& Marzi, 2016).

The visual N1 is the first negative peak after P1, widely distributed over the entire scalp but peaking at frontal regions before posterior. Based on this and on studies such as (Vogel \& Luck, 2000), it is suggested that visual $\mathrm{N} 1$ is related to a discrimination process within the focal area of attention.

The visual N2pc is a very well studied component related to selective attention, known to appear posterior-contralateral to the focus of attention. In this case the PSICAT protocol presents stimuli centrally and N2pc is not elicited systematically.

The P3 is among the most widely studied components, a positive wave appearing between 250 to 500ms (B. Cowley, Filetti, et al., 2016). The P3 is an endogenous component independent of the physical properties of the eliciting stimulus, implying it is related to decision making and attention. P3 comprises two sub-components, P3a and P3b, which index separate cognitive processes related to attention (Polich, 2007). The P3a shows largest amplitude over frontal/central electrode sites from 250-280ms; whereas the P3b is maximal in parietal areas from $300-500 \mathrm{~ms}$. P3a and P3b also show distinct patterns of amplitude, latency and neuropharmacology (Polich, 2007). John 
Polich proposes an explanatory model where P3 represents an early attention process: first an orienting change to a frontal working memory representations produces the P3a; this attention-driven stimulus signal then passes to temporal-parietal structures to create the P3b (Polich, 2007).

Against this background, the ERP results validate expectations, similarly to behavioural results albeit with somewhat more complexity. Questions of congruency are certainly strongly answered, with very large ES from the contrasts in P3a,b (see Figure 4). The scalp map for congruent P3a also indicates that this condition has a strong left-hemisphere bias, unlike other conditions. This seems to contradict earlier work (Sokhadze et al., 2012; Wasserstein et al., 1987), which however studied clinical samples. In healthy individuals, it is possible that SCI classification becomes a categorical task when defined in a bounded context such as this experimental protocol, and thus processed in left cortex (Marsolek, 1995); rather than the right-hemisphere localised gestalt integration task one would expect. The scalp map for incongruent P3a conversely shows strong occipital negativation, indicating the processing of novel visual stimuli.

N1 in response to primers follows hypothesis H4pN1 (and (Marini \& Marzi, 2016)), i.e. pri.SCI.N1 < pri.noSCI.N1; with large ES. However, the early components after target onset are less clearly enhanced by SCIs. This seems to be due to the primers, as follows. Although incongruent SCI has more negative N1 than incongruent no-SCI, there is a close morphological match of incongruent SCI to congruent no-SCI from $-100 \ldots 200 \mathrm{~ms}$ (see Figure 5, panel A, right plot), and these latter conditions share a no-SCI primer. This suggests that for these conditions, the no-SCI primer is the main influence on neural processing until after the target N1. Such a view accords with the thesis of (Marini \& Marzi, 2016) and the result for H4pN1, that SCI primer shapes capture attention and are processed earlier, and consequently are overridden by following targets at an earlier time compared to no-SCI primers. The P3a results for $\mathbf{H 4 t P 3}$ are only marginally significant but visually show clear differentiation, see Figure 5, panel B. They are consistent across conditions, suggesting that the effect of primers is no longer dominant at this latency. This view is supported by examining the difference curves, (Figure 5, panel A bottom), where the black curve for congruent SCI, no-SCI difference is closely mirrored until $300 \mathrm{~ms}$ by the inverse incongruent curve; i.e. the incongruent primer SCI, no-SCI difference.

\subsubsection{Neural correlates of sustained attention}

Sustained attention involves cooperation of a number of anatomical areas. For example, upon detection of inadequate attentional focus the posterior me- 
dial frontal cortex (pMFC) sends a signal via lateral prefrontal cortex (LPFC) to lower level sensory areas (Clayton et al., 2015). pMFC includes the anterior cingulate cortex (ACC) and dorsomedial prefrontal cortex (dmPFC).

Such anatomical inter-areal communication during sustained attention indicates the role of cortical oscillations. An explanatory model is given by (Clayton et al., 2015), where fronto-medial theta (4-8 Hz) power is suggested to reflect cognitive control processes, which monitor errors due to attentional lapses. These control processes are seen as fronto-posterior power-phase coupling to promote localised gamma oscillations $(30-100 \mathrm{~Hz})$ in task-relevant areas, and alpha (8-12 Hz) oscillations in task-irrelevant areas.

However others have suggested that fronto-medial theta reflects conditions of high working memory load (Klimesch, 1999); or mental fatigue (Wascher et al., 2014). Indeed, fronto-medial theta power tends to increase across the duration of sustained attention tasks. This increase correlates with more commission errors and slowing of post-error RTs (Wascher et al., 2014): all suggestive of fatigue or excessive load (Holm, Lukander, Korpela, Sallinen, \& Müller, 2009).

Thus fronto-medial theta is both associated with cognitive control and also increased by fatigue and error. This apparent contradiction is addressed by (Clayton et al., 2015), who suggests that fronto-medial theta responds to detection of inattention errors by attempting to compensate with increased signalling; to which however fatigue-depleted networks may be unable to respond.

(Clayton et al., 2015) follows (Klimesch, 1999) in suggesting that increased oscillatory alpha power in specific sensory areas is indicative of inhibition of task-irrelevant areas. However another view was proposed by (Palva \& Palva, 2007), in which the observed alpha increase plays a direct role in sustaining mental representations, through mechanisms of power-phase coupling within the fronto-parietal network $(\mathrm{FPN})$. Under this view, supposedly task-irrelevant areas are in fact supportive of the task, which reduces the need to categorise large areas of cortex as 'task-irrelevant' and in fact enriches the framework from (Clayton et al., 2015).

The results for sustained attention analysis are thus as expected: theta power is elevated in the last $10 \%$ of trials, compared to the first $10 \%$ (Figure 6 , panel A). ERSP indicates that this elevation most strongly affects first $\sim 60 \mathrm{~ms}$ post-target onset (Figure 6, panel B), dampening the event-locked power increase over baseline. The strong phase-locking at $5-7 \mathrm{~Hz}$ in early trials (Figure 6 , panel D, left plot showing $\sim 0.27$ ITC), is quite diminished by late trials, significantly $(p<0.01)$ after $\sim 200 \mathrm{~ms}$. This implies diminishment of attending (Yamagishi et al., 2008) in late trials, which in turn suggests that FM theta-band power does not increase due to, e.g. working memory load 
(Klimesch, 1999), but due to genuine mental fatigue (Holm et al., 2009). The ERP image (Figure 6, panel $\mathrm{C}$ ) also indicates that event-locked amplitude diminishes from early to late trials, quite consistently across all trials in each subset.

\subsection{Congruency vs. target-condition}

The final result on relative ES of congruency and (no-)SCI questions is surprisingly strong. The notion that intra-modal interference demands more neural processing than a clearly defined target has serious implications for the way attention is engaged in, for example, online search or TechnologyEnhanced Learning (TEL) software. The impact of distractors on attention, including for clinical studies of ADHD, has tended to be examined with multimodal protocols, e.g. visual targets and auditory distractors (Gumenyuk et al., 2005). Therefore, not only have I shown (for the first time to my knowledge) that the congruency of intra-modal primers is a stronger stimulus than the task-defined target; also PSICAT offers a novel open-source tool to probe such effects in valuable clinical/societal questions.

\subsection{Other work/future work}

\subsubsection{Protocols}

Sustained attention is usually tested with infrequent-target continuous performance tests, such as Integrated Visual and Auditory CPT (IVA-2), Conners' CPT-II, and T.O.V.A. (Fortenbaugh et al., 2017). Designed to detect the presence of attentional problems typical for ADHD, T.O.V.A. includes both infrequent and frequent target conditions which makes it particularly interesting as the condition effects can be contrasted and consequently, the EEG correlates of T.O.V.A. have been studied (Juurmaa, 2017) (as has Conners' CPT Loo et al). In general, such clinical CPTs are non-optimal as labratory protocols to probe integrated cognition, because of the aforementioned simplest-possible stimulus design, and due to lack of facility to balance conditions across a sample.

Gestalt imagery is used in a number of clinical neuropsychological tests, but the emphasis is largely on testing peceptual function ${ }^{4}$. A notable test of this kind is L-POST (Torfs, Vancleef, Lafosse, Wagemans, \& De-Wit, 2014) which is computerised, freely available, and allows access to raw data.

\footnotetext{
${ }^{4} \mathrm{An}$ overview of such tests is maintained at http://www.gestaltrevision.be/en/ master-index/83-resources/reference-guides/
} 
Thus, although many papers have studied the perceptual properties of gestalt imagery (showing that Kanizsa SCIs require awareness (Banica \& Schwarzkopf, 2016) and are thus good for attention testing), few publications have used Kanizsa SCIs to study attention. In one example, (Sokhadze et al., 2012) used Kanizsa SCIs to test the ERPs of ADHD children, finding delayed early components in response to target stimuli and also that the delayed P3a component was better represented in the right hemisphere. However, code has not been released for any published protocol, to my knowledge.

\subsubsection{Future work}

PSICAT was originally designed and deployed in context of a clinical trial. The next PSICAT publication will report this work, comparing the healthy controls analysed herein to the ADHD group. The ADHD group is expected to perform worse on incongruent trials compared to controls, because of strong evidence for deficient saliency processing in ADHD individuals (Cubillo, Halari, Giampietro, Taylor, \& Rubia, 2011). On the other hand such results might be explained by the neuroenergetics hypothesis (Killeen, Russell, \& Sergeant, 2013), leading over time to reduced inhibition of interference. Untangling such questions with PSICAT data will be a valuable addition to the literature.

In a broader view, prior work has pointed out a 'lacuna' in our understanding of integrated higher cognition (Logie, Trawley, \& Law, 2011). PSICAT is a step toward improving that situation. Application in more studies, with a range of brain imaging techniques or integration to clinical trials, will generate interesting novel insights.

In such applications, various parameters of the protocol might be manipulated to test specific questions. For example, the ITI in PSICAT is rather short at $4-600 \mathrm{~ms}$. Increasing the ITI to tens of seconds would allows the protocol to test vigilant responding. The congruency between matched primers and SCIs is quite strong because there is no visual mask between them. Various types of masks could be used to probe the conditions under which the large RT advantage for the con.SCI condition (Figure 3) is decreased.

Technically, the code for PSICAT could ideally be ported to a free and non-propietary platform such as Python, to support the democratization of research.

\subsection{Conclusion}

Gestalt imagery, including Kanizsa shapes, is a class of visual stimuli that enables perceptual inference of implied images. Thus gestalt perception log- 
ically involves a more extensive integrative network than would perception of similar visual features with no gestalt property (as has also been shown empirically, see section 4.1). For this reason I designed the PSICAT protocol based on the presentation of Kanizsa stimuli targets, built from Pac-Manlike shapes that induce an SCI if angled collinearly. Targets are preceded by acute-angle line primers.

The PSICAT protocol uses primer-target stimuli pairs which are either congruent and saliency reinforcing, or incongruent and task-interfering. The congruency of primers modulates the saliency of edges in the SCI. Being task-irrelevant, primers constitute a case of target-interference. When the interference is presented congruently, the primer contours evoke the same processing response as the following target stimulus. Thus the saliency is reinforced. When incongruent, the primer clearly reinforces the opposite class to the target stimulus and induce a requirement for interference inhibition.

While extending the state of the art for CPTs of sustained attention, the PSICAT protocol aims to remain as simple as possible to prevent unwanted confounds; but it is open to customization. Open-source code hosted on a public repository (https://github.com/zenBen/Kanizsa_Prime/) makes it possible to freely use or adapt the PSICAT functionality, including the capacity to generate geometrically defined SCI shapes in Neurobs Presentation format. This can serve as the basis for entirely new protocol designs.

Behavioural and neural results have clearly validated the design of the protocol, and the open-source publication of the protocol code and resources render this a valuable tool for future research on integrative neural networks for processing of visual attention, in basic and clinical settings.

\section{Acknowledgements}

I wish to thank Kristiina Juurmaa for assistance during programming and data gathering, plus comments to the text; also Otto Lappi, Kristian Lukander and Miika Leminen for valuable feedback on drafts of the manuscript.

This work was partly funded by TEKES, the Finnish national funding body, under grant \#440078. 


\section{References}

Banica, T., \& Schwarzkopf, D. S. (2016). Induction of kanizsa contours requires awareness of the inducing context. PLOS ONE, 11, 1-20. doi: 10.1371/journal.pone. 0161177

CHADD. (2017). Children and Adults with Attention-Deficit/Hyperactivity Disorder: National Resource on ADHD. Retrieved 13.08.2017, from http://www. chadd.org/Understanding-ADHD/About-ADHD/ Data-and-Statistics/General-Prevalence.aspx

Clayton, M. S., Yeung, N., \& Cohen Kadosh, R. (2015). The roles of cortical oscillations in sustained attention. Trends in Cognitive Sciences, 19(4), 188-195. doi: 10.1016/j.tics.2015.02.004

Cowley, B., Filetti, M., Lukander, K., Torniainen, J., Henelius, A., Ahonen, L., ... Jaccuci, G. (2016). The Psychophysiology Primer: a guide to methods and a broad review with a focus on human computer interaction. Foundations and Trends in HCI, 9(3-4), 151-308. doi: $10.1561 / 1100000065$

Cowley, B., Holmström, É., Juurmaa, K., Kovarskis, L., \& Krause, C. M. (2016). Computer Enabled Neuroplasticity Treatment: A Clinical Trial of a Novel Design for Neurofeedback Therapy in Adult ADHD. Frontiers in Human Neuroscience, $10(205)$.

Cowley, B., \& Lukander, K. (2016). Forest, Trees, Dynamics: Results from a Wisconsin Card Sorting Test variant Protocol for Studying GlobalLocal Attention and Complex Cognitive Processes. Frontiers in Psychology: Cognitive Science, 7(238).

Cowley, B. U., Korpela, J., \& Torniainen, J. (2017, March). Computational testing for automated preprocessing: a matlab toolbox to enable large scale electroencephalography data processing. PeerJ Computer Science, 3, e108. doi: 10.7717/peerj-cs.108

Cubillo, A., Halari, R., Giampietro, V., Taylor, E., \& Rubia, K. (2011). Fronto-striatal underactivation during interference inhibition and attention allocation in grown up children with attention deficit/hyperactivity disorder and persistent symptoms. Psychiatry research, 193(1), 17-27. doi: 10.1016/j.pscychresns.2010.12.014

Delorme, A., \& Makeig, S. (2004). EEGLAB: an open source toolbox for analysis of single-trial EEG dynamics including independent component analysis. Journal of neuroscience methods, 134(1), 9-21. doi: 10.1016/j.jneumeth.2003.10.009

Delorme, A., Mullen, T., Kothe, C., Akalin Acar, Z., Bigdely-Shamlo, N., Vankov, A., \& Makeig, S. (2011). EEGLAB, SIFT, NFT, BCILAB, and ERICA: new tools for advanced EEG processing. Computational intel- 
ligence and neuroscience, 2011, 130714. doi: 10.1155/2011/130714

Field, A. (2009). Discovering statistics using SPSS (and sex, drugs and rock 'n' roll) (3rd ed.). London, UK: Sage Publications.

Fortenbaugh, F. C., DeGutis, J., \& Esterman, M. (2017, May). Recent theoretical, neural, and clinical advances in sustained attention research. Annals of the New York Academy of Sciences, 1396(1), 70-91. doi: $10.1111 /$ nyas. 13318

Gumenyuk, V., Korzyukov, O., Escera, C., Hämäläinen, M., Huotilainen, M., Häyrinen, T., ... Alho, K. (2005, mar). Electrophysiological evidence of enhanced distractibility in ADHD children. Neuroscience letters, $374(3), 212-7$.

Häkkinen, J., \& Nyman, G. (2001). Phantom surface captures stereopsis. Vision Research, 41(2), 187-199. doi: 10.1016/S0042-6989(00)00231 $-5$

Harris, J. J., Schwarzkopf, D. S., Song, C., Bahrami, B., \& Rees, G. (2011). Contextual illusions reveal the limit of unconscious visual processing. Psychological science, 22(3), 399-405. doi: 10.1177/ 0956797611399293

Hellige, J. (1993). Hemispheric asymmetry : what's right and what's left. Cambridge Mass.: Harvard University Press.

Holm, A., Lukander, K., Korpela, J., Sallinen, M., \& Müller, K. M. I. (2009, Jul). Estimating Brain Load from the EEG. The Scientific World JOURNAL, 9, 639-651. doi: 10.1100/tsw.2009.83

Itti, L., \& Koch, C. (2001). Computational modelling of visual attention. Nature reviews. Neuroscience, 2(3), 194-203. doi: 10.1038/35058500

Juurmaa, K. (2017). Alpha and Theta Oscillations during Sustained Attention in Adults with Attention-Deficit Hyperactivity Disorder (Unpublished master's thesis). University of Helsinki, Helsinki, Finland.

Killeen, P. R., Russell, V. A., \& Sergeant, J. A. (2013). A behavioral neuroenergetics theory of ADHD. Neuroscience and biobehavioral reviews, 37(4), 625-57. doi: 10.1016/j.neubiorev.2013.02.011

Klimesch, W. (1999). EEG alpha and theta oscillations reflect cognitive and memory performance: a review and analysis. Brain research. Brain research reviews., 29(2), 169.

Kogo, N., Strecha, C., Van Gool, L., \& Wagemans, J. (2010). Surface construction by a 2-D differentiation-integration process: A neurocomputational model for perceived border ownership, depth, and lightness in Kanizsa figures. Psychological Review, 117(2), 406-439. doi: 10.1037/a0019076

Lee, T. W., Girolami, M., \& Sejnowski, T. J. (1999, Feb). Independent component analysis using an extended infomax algorithm for mixed 
subgaussian and supergaussian sources. Neural computation, 11(2), 417-41.

Logie, R. H., Trawley, S., \& Law, A. (2011, Nov). Multitasking: multiple, domain-specific cognitive functions in a virtual environment. Memory Ef cognition, 39(8), 1561-74. doi: 10.3758/s13421-011-0120-1

Loo, S. K., Hale, T. S., Macion, J., Hanada, G., McGough, J. J., McCracken, J. T., \& Smalley, S. L. (2009). Cortical activity patterns in ADHD during arousal, activation and sustained attention. Neuropsychologia, 47(10), 2114-2119. doi: 10.1016/j.neuropsychologia.2009.04.013

Luck, S. J. (2014). An Introduction to the Event-Related Potential Technique (2nd ed.). Cambridge MA: MIT Press.

Marini, F., \& Marzi, C. A. (2016). Gestalt Perceptual Organization of Visual Stimuli Captures Attention Automatically: Electrophysiological Evidence. Frontiers in Human Neuroscience, 10, 446. doi: 10.3389/ fnhum.2016.00446

Marsolek, C. J. (1995). Abstract visual-form representations in the left cerebral hemisphere. (Vol. 21) (No. 2). US: American Psychological Association. doi: 10.1037/0096-1523.21.2.375

Mognon, A., Jovicich, J., Bruzzone, L., \& Buiatti, M. (2011, Feb). ADJUST: An automatic EEG artifact detector based on the joint use of spatial and temporal features. Psychophysiology, 48(2), 229-240. doi: 10 $.1111 / \mathrm{j} .1469-8986.2010 .01061 . x$

Murray, M. M., Wylie, G. R., Higgins, B. A., Javitt, D. C., Schroeder, C. E., \& Foxe, J. J. (2002). The spatiotemporal dynamics of illusory contour processing: combined high-density electrical mapping, source analysis, and functional magnetic resonance imaging. The Journal of neuroscience : the official journal of the Society for Neuroscience, 22(12), $5055-73$.

Newport, C. (2016). Deep work : rules for focused success in a distracted world. New York: Grand Central Publishing.

Nolan, H., Whelan, R., \& Reilly, R. B. (2010, Sep). FASTER: Fully Automated Statistical Thresholding for EEG artifact Rejection. Journal of Neuroscience Methods, 192(1), 152-162. doi: 10.1016/j.jneumeth.2010 .07 .015

Palva, S., \& Palva, J. M. (2007). New vistas for $\alpha$-frequency band oscillations. Trends in Neurosciences, 30(4), 150-158. doi: 10.1016/j.tins.2007.02 .001

Polich, J. (2007). Updating P300: an integrative theory of P3a and P3b. Clinical neurophysiology : official journal of the International Federation of Clinical Neurophysiology, 118(10), 2128-48. doi: 10.1016/ j.clinph.2007.04.019 
R Core Team. (2014). R: A language and environment for statistical computing [Computer software manual]. Vienna, Austria. Retrieved from http://www.R-project.org/

Re, A. C. D. (2013). compute.es: Compute effect sizes [Computer software manual]. Retrieved from http://cran.r-project.org/web/ packages/compute.es

Ringach, D. L., \& Shapley, R. (1996). Spatial and Temporal Properties of Illusory Contours and Amodal Boundary Completion. Vision Research, 36 (19), 3037-3050. doi: 10.1016/0042-6989(96)00062-4

Robertson, I. H., \& O'Connell, R. (2010). Vigilant attention. In A. C. Nobre \& J. T. Coull (Eds.), Attention and time (pp. 79-88). OUP Oxford.

Sawilowsky, S. S. (2009). New Effect Size Rules of Thumb. Journal of Modern Applied Statistical Methods, 8(2), 597-599. doi: 10.22237/ jmasm/1257035100

Schurger, A., Sarigiannidis, I., Naccache, L., Sitt, J. D., \& Dehaene, S. (2015). Cortical activity is more stable when sensory stimuli are consciously perceived. Proceedings of the National Academy of Sciences of the United States of America, 112(16), E2083-92. doi: 10.1073/ pnas. 1418730112

Schwarzkopf, D. S., \& Rees, G. (2011). Interpreting local visual features as a global shape requires awareness. Proceedings. Biological sciences, 278(1715), 2207-15. doi: 10.1098/rspb.2010.1909

Sokhadze, E. M., Baruth, J. M., Sears, L., Sokhadze, G. E., El-Baz, A. S., Williams, E., .. Casanova, M. F. (2012). Event-related potential study of attention regulation during illusory figure categorization task in ADHD, autism spectrum disorder, and typical children. Journal of neurotherapy, 16(1), 12-31. doi: 10.1080/10874208.2012.650119

Tanskanen, T., Saarinen, J., Parkkonen, L., \& Hari, R. (2008). From local to global: Cortical dynamics of contour integration. Journal of Vision, 8(7), 15. doi: $10.1167 / 8.7 .15$

Torfs, K., Vancleef, K., Lafosse, C., Wagemans, J., \& De-Wit, L. (2014). The Leuven Perceptual Organization Screening Test (L-POST), an online test to assess mid-level visual perception. Behavior Research Methods, 46(2), 472-487. doi: 10.3758/s13428-013-0382-6

Vogel, E. K., \& Luck, S. J. (2000). The visual N1 component as an index of a discrimination process. Psychophysiology, 37(2), 190-203.

Wagemans, J., Elder, J. H., Kubovy, M., Palmer, S. E., Peterson, M. A., Singh, M., \& von der Heydt, R. (2012, Nov). A century of Gestalt psychology in visual perception: I. Perceptual grouping and figureground organization. Psychological bulletin, 138(6), 1172-217. doi: $10.1037 / \mathrm{a} 0029333$ 
Wagemans, J., Feldman, J., Gepshtein, S., Kimchi, R., Pomerantz, J. R., van der Helm, P. A., \& van Leeuwen, C. (2012). A century of Gestalt psychology in visual perception: II. Conceptual and theoretical foundations. Psychological bulletin, 138(6), 1218-52. doi: 10.1037/a0029334

Wascher, E., Rasch, B., Sänger, J., Hoffmann, S., Schneider, D., Rinkenauer, G., ... Gutberlet, I. (2014). Frontal theta activity reflects distinct aspects of mental fatigue. Biological Psychology, 96, 57-65. doi: 10 .1016/j.biopsycho.2013.11.010

Wasserstein, J., Zappulla, R., Rosen, J., Gerstman, L., \& Rock, D. (1987). In search of closure: subjective contour illusions, Gestalt completion tests, and implications. Brain and cognition, 6(1), 1-14.

Whelan, R. (2010). Effective analysis of reaction time data. The Psychological Record, 58(3), 475-482.

Wolfe, J. M., \& Horowitz, T. S. (2004). What attributes guide the deployment of visual attention and how do they do it? Nature reviews. Neuroscience, 5(6), 495-501. doi: 10.1038/nrn1411

Woodman, G. F. (2010). A brief introduction to the use of event-related potentials in studies of perception and attention. Attention, perception \& psychophysics, 72 (8), 2031-46. doi: 10.3758/APP.72.8.2031

Yamagishi, N., Callan, D. E., Anderson, S. J., \& Kawato, M. (2008, mar). Attentional changes in pre-stimulus oscillatory activity within early visual cortex are predictive of human visual performance. Brain Research, 1197, 115-122. doi: 10.1016/j.brainres.2007.12.063 